
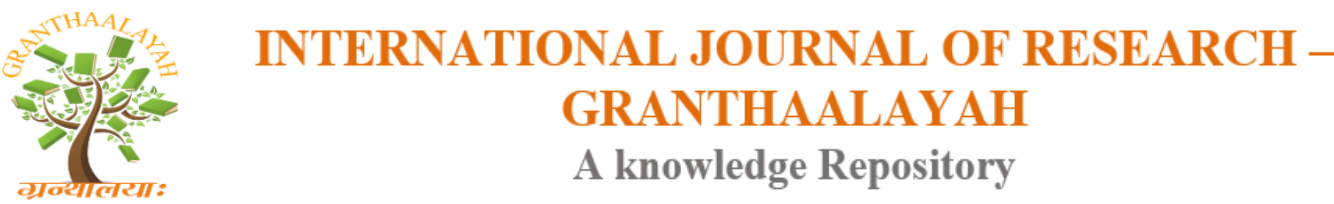

Science

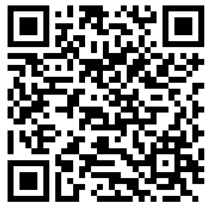

\title{
ENRICHED CUCKOO ALGORITHM FOR ACTIVE POWER LOSS REDUCTION
}

\author{
Dr.K.Lenin *1 \\ ${ }^{* 1}$ Professor, Department of EEE, Prasad V.Potluri Siddhartha Institute of Technology, Kanuru, \\ Vijayawada, Andhra Pradesh -520007, India
}

\begin{abstract}
This paper proposes Group Competition (GC) algorithm for solving optimal reactive power problem. Group Competition (GC) algorithm stimulated from the contest of sport teams in a sport group. A number of individuals as sport teams contend in a simulated group for numerous weeks. Based on the group schedule in every week, teams play in pairs and their game result is determined in terms of win or loss, given known the playing strength along with the teams' planned formations. Modeling an artificial match analysis, each team devises a new playing strategy for the subsequent week competition and this procedure is repetitive for number of seasons. In order to evaluate the validity of the proposed Group Competition (GC) algorithm, it has been tested on Standard IEEE 57,118 bus systems and simulation results reveal about the good performance of the proposed algorithm in reducing real power loss and voltage profiles are within the limits.
\end{abstract}

Keywords: Optimal Reactive Power; Transmission Loss; Cuckoo Search Algorithm; Global Optimization; Lévy Flight.

Cite This Article: Dr.K.Lenin. (2017). "ENRICHED CUCKOO ALGORITHM FOR ACTIVE POWER LOSS REDUCTION." International Journal of Research - Granthaalayah, 5(11), 307-315. https://doi.org/10.29121/granthaalayah.v5.i11.2017.2357.

\section{Introduction}

Optimal reactive power dispatch problem is one of the difficult optimization problems in power systems. The sources of the reactive power are the generators, synchronous condensers, capacitors, static compensators and tap changing transformers. The problem that has to be solved in a reactive power optimization is to determine the required reactive generation at various locations so as to optimize the objective function. Here the optimal reactive power problem involves best utilization of the existing generator bus voltage magnitudes, transformer tap setting and the output of reactive power sources so as to minimize the real power loss and to keep the voltage profiles within the limits. Various mathematical techniques have been adopted to solve this optimal reactive power dispatch problem. These include the gradient method [1-2], Newton method [3] and linear programming [4-7].The gradient and Newton methods suffer from the 
difficulty in handling inequality constraints. To apply linear programming, the input- output function is to be expressed as a set of linear functions which may lead to loss of accuracy. Recently global Optimization techniques such as genetic algorithms have been proposed to solve the reactive power flow problem [8,9]. This paper presents an Enriched Cuckoo Search (ECS) algorithm for solving optimal reactive power problem. The cuckoo search (CS) algorithm is a recently developed algorithm [10-12], which has been applied to different types of optimization problem. Due to the parameters of the cuckoo search algorithm has been kept constant \& it decreases the efficiency of the algorithm. In this paper cuckoo search algorithm parameters pa, $\lambda$ and $\alpha$ are tuned to find improved solutions. In order to evaluate the performance of the proposed algorithm, it has been tested in Standard IEEE 57,118 bus systems and compared to other standard reported algorithms. Simulation results show's that Enriched Cuckoo Search (ECS) algorithm successfully reduces the real power loss and voltage profiles are within the limits.

\section{Problem Formulation}

\subsection{Active Power Loss}

The objective of the reactive power dispatch is to minimize the active power loss in the transmission network, which can be described as follows:

$F=P L=\sum_{k \in N b r} g_{k}\left(V_{i}^{2}+V_{j}^{2}-2 V_{i} V_{j} \cos \theta_{i j}\right)$

Where F- objective function, $\mathrm{P}_{\mathrm{L}}$ - power loss, $\mathrm{g}_{\mathrm{k}}$-conductance of branch, $\mathrm{V}_{\mathrm{i}}$ and $\mathrm{V}_{\mathrm{j}}$ are voltages at buses $\mathrm{i}, \mathrm{j}, \mathrm{Nbr}$ - total number of transmission lines in power systems.

\subsection{Voltage Profile Improvement}

For minimizing the voltage deviation in PQ buses, the objective function becomes:

$F=P L+\omega_{v} \times V D$

Where VD - voltage deviation, $\omega_{\mathrm{v}}$ - is a weighting factor of voltage deviation.

Voltage deviation given by:

$V D=\sum_{i=1}^{N p q}\left|V_{i}-1\right|$

Where Npq- number of load buses

\subsection{Equality Constraint}

The equality constraint of the problem is represented by the power balance equation, where the total power generation must cover the total power demand and the power losses:

$P_{G}=P_{D}+P_{L}$ 
Where $\mathrm{P}_{\mathrm{G}}$ - total power generation, $\mathrm{P}_{\mathrm{D}}$ - total power demand.

\subsection{Inequality Constraints}

The inequality constraints in the power system as well as the limits created to ensure system security. Upper and lower bounds on the active power of slack bus (Pg), and reactive power of generators $\left(\mathrm{Q}_{\mathrm{g}}\right)$ are written in mathematically as follows:

$P_{\text {gslack }}^{\min } \leq P_{\text {gslack }} \leq P_{\text {gslack }}^{\max }$

$Q_{g i}^{\min } \leq Q_{g i} \leq Q_{g i}^{\max }, i \in N_{g}$

Upper and lower bounds on the bus voltage magnitudes $\left(\mathrm{V}_{\mathrm{i}}\right)$ :

$V_{i}^{\min } \leq V_{i} \leq V_{i}^{\max }, i \in N$

Upper and lower bounds on the transformers tap ratios $\left(\mathrm{T}_{\mathrm{i}}\right)$ :

$T_{i}^{\min } \leq T_{i} \leq T_{i}^{\max }, i \in N_{T}$

Upper and lower bounds on the compensators reactive powers $\left(\mathrm{Q}_{\mathrm{c}}\right)$ :

$Q_{C}^{\min } \leq Q_{c} \leq Q_{C}^{\max }, i \in N_{C}$

Where $\mathrm{N}$ is the total number of buses, $\mathrm{N}_{\mathrm{T}}$ is the total number of Transformers; $\mathrm{N}_{\mathrm{c}}$ is the total number of shunt reactive compensators.

\section{Cuckoo Search Algorithm (Cs)}

The cuckoo search algorithm (CS) was inspired by the obligate brood parasitism of some cuckoo species by laying their eggs in the nests of host birds. Some cuckoos have evolved in such a way that female parasitic cuckoos can imitate the colours and patterns of the eggs of a few chosen host species. This reduces the probability of the eggs being abandoned and, therefore, increases their re-productivity. In general, the cuckoo eggs hatch slightly earlier than their host eggs. Once the first cuckoo chick is hatched, his first instinct action is to evict the host eggs by blindly propelling the eggs out of the nest. This action results in increasing the cuckoo chick's share of food provided by its host bird. Moreover, studies show that a cuckoo chick can imitate the call of host chicks to gain access to more feeding opportunity. The CS models such breeding behavior and, thus, can be applied to various optimization problems. The performance of the CS can be improved by using Lévy Flights instead of simple random walk.

\subsection{Lévy Flights}

In nature, animals search for food in a random or quasi random manner. Generally, the foraging path of an animal is effectively a random walk because the next move is based on both the current location/state and the transition probability to the next location. The chosen direction 
implicitly depends on a probability, which can be modeled mathematically. Various studies have shown that the flight behavior of many animals and insects demonstrates the typical characteristics of Lévy flights [13]. A Lévy flight is a arbitrary walk in which the step-lengths are distributed according to a heavy-tailed probability distribution. After a large number of steps, the distance from the origin of the random walk tends to a stable distribution.

\subsection{Cuckoo Search Implementation}

Each egg in a nest represents a solution, and a cuckoo egg represents a new solution. The aim is to employ the new and potentially better solutions (cuckoos) to replace not-so-good solutions in the nests. In the simplest form, each nest has one egg. The algorithm can be extended to more complicated cases in which each nest has multiple eggs representing a set of solutions. The cuckoo search algorithm (CS) is based on three idealized rules:

a) Each cuckoo lays one egg at a time, and dumps it in a randomly chosen nest;

b) The best nests with high quality of eggs (solutions) will carry over to the next generations;

c) The number of available host nests is fixed, and a host can discover an alien egg with probability $\mathrm{p}_{\mathrm{a}} \epsilon[0,1]$ In this case, the host bird can either throw the egg away or abandon the nest to build a completely new nest in a new location.

For simplicity, the last assumption can be approximated by a fraction pa of the $\mathrm{n}$ nests being replaced by new nests, having new random solutions. For a maximization problem, the quality or fitness of a solution can simply be proportional to the objective function. Other forms of fitness can be defined in a similar way to the fitness function in genetic algorithms.

The basic steps of the cuckoo search algorithm (CS) can be summarized as follows,

Begin

Objective function $f(x), x=\left(x_{1, \ldots \ldots .,} x_{d}\right)^{T}$

Generate initial population of $n$ host nests $x_{i},(i=1,2, . ., n)$

While (t Max Generation) or (stop criterion)

Get a cuckoo randomly by Lévy flights

Evaluate its quality / fitness $\mathrm{F}_{\mathrm{i}}$

Choose a nest among $n$ (say.j) randomly

If $\left(F_{i}>F_{j}\right)$ Replace $j$ by the new solution;

End if

A fraction $\left(\mathrm{p}_{\mathrm{a}}\right)$ of worse nests are abandoned and new ones are built;

Keep the best solutions (or nests with quality solutions);

Rank the solutions and find the current best

End while

Post process results and visualization

End

When generating new solutions $x_{i}(t+1)$ for the ith cuckoo, the following Lévy flight is performed 
$x_{i}(t+1)=x_{i}(t)+\alpha \Theta \operatorname{Levy}(\lambda)$

Where $\alpha>0$ is the step size, which should be related to the scale of the problem of interest. The product $\Theta$ means entry-wise multiplications. In this research work, we consider a Lévy flight in which the step-lengths are distributed according to the following probability distribution

Levy $u=t^{-1}, 1<\lambda \leq 3$

Here, the consecutive jumps/steps of a cuckoo essentially form a random walk process which obeys a power-law step-length distribution with a heavy tail. It is worth pointing out that, in the real world, if a cuckoo's egg is very similar to a host's eggs, then this cuckoo's egg is less likely to be discovered, thus the fitness should be related to the difference in solutions. Therefore, it is a good idea to do a random walk in a biased way with some random step sizes.

\section{Enriched Cuckoo Search (ECS) Algorithm}

The parameters pa, $\lambda$ and $\alpha$ introduced in the cuckoo search algorithm (CS) help the algorithm to find globally and locally improved solutions, respectively. The parameters pa and $\alpha$ are very important parameters in fine-tuning of solution vectors, and can be potentially used in adjusting convergence rate of algorithm. The traditional cuckoo search algorithm (CS) uses fixed value for both pa and $\alpha$. These values are set in the initialization step and cannot be changed during new generations. The main drawback of this method appears in the number of iterations to find an optimal solution. If the value of pa is small and the value of $\alpha$ is large, the performance of the algorithm will be poor and leads to considerable increase in number of iterations. If the value of pa is large and the value of $\alpha$ is small, the speed of convergence is high but it may be unable to find the best solutions.

The key difference between the Enriched Cuckoo Search (ECS) algorithm and cuckoo search algorithm (CS) is in the way of adjusting pa and $\alpha$. To improve the performance of the CS algorithm and eliminate the drawbacks lies with fixed values of pa and $\alpha$, the ECS algorithm uses variables pa and $\alpha$. In the early generations, the values of pa and $\alpha$ must be big enough to enforce the algorithm to increase the diversity of solution vectors. However, these values should be decreased in final generations to result in a better fine-tuning of solution vectors. The values of pa and $\alpha$ are dynamically changed with the number of generation and expressed in Equations (12- 14), where NI and gn are the number of total iterations and the current iteration, respectively.

$$
\begin{aligned}
& p_{a}(g n)=p_{a \max }-\frac{g n}{N I}\left(p_{a \max }-p_{a \min }\right) \\
& \alpha(g n)=\alpha_{\max } \exp (c . g n) \\
& c=\frac{1}{N I} \operatorname{Ln}\left(\frac{\alpha_{\min }}{\alpha_{\max }}\right)
\end{aligned}
$$




\section{Simulation Results}

At first Enriched Cuckoo Search (ECS) algorithm has been tested in standard IEEE-57 bus power system. The reactive power compensation buses are 18, 25 and 53. Bus 2, 3, 6, 8, 9 and 12 are PV buses and bus 1 is selected as slack-bus. The system variable limits are given in Table 1 . The preliminary conditions for the IEEE-57 bus power system are given as follows:

$\mathrm{P}_{\text {load }}=12.129$ p.u. Q $_{\text {load }}=3.092$ p.u.

The total initial generations and power losses are obtained as follows:

$\sum P_{G}=12.484$ p.u. $\sum Q_{G}=3.3193$ p.u.

$\mathrm{P}_{\text {loss }}=0.25867$ p.u. $\mathrm{Q}_{\text {loss }}=-1.2078$ p.u.

Table 2 shows the various system control variables i.e. generator bus voltages, shunt capacitances and transformer tap settings obtained after optimization which are within the acceptable limits. In Table 3, shows the comparison of optimum results obtained from proposed methods with other optimization techniques. These results indicate the robustness of proposed approaches for providing better optimal solution in case of IEEE-57 bus system.

Table 1: Variable Limits

\begin{tabular}{|c|c|c|c|c|c|c|c|c|}
\hline \multicolumn{9}{|c|}{ Reactive Power Generation Limits } \\
\hline Bus no & 1 & \multicolumn{2}{|l|}{2} & 3 & 6 & 8 & 9 & 12 \\
\hline Qgmin & -1.4 & \multicolumn{2}{|c|}{-.015} & -.02 & -0.04 & -1 & -0.03 & -0.4 \\
\hline Qgmax & 1 & \multicolumn{2}{|c|}{0.3} & 0.4 & 0.21 & 1 & 0.04 & 1.50 \\
\hline \multicolumn{9}{|c|}{ Voltage And Tap Setting Limits } \\
\hline vgmin & \multicolumn{2}{|c|}{ Vgmax } & \multicolumn{2}{|c|}{ vpqmin } & \multicolumn{2}{|c|}{ Vpqmax } & tkmin & tkmax \\
\hline 0.9 & \multicolumn{2}{|c|}{1.0} & \multicolumn{2}{|c|}{0.91} & \multicolumn{2}{|l|}{1.05} & 0.9 & 1.0 \\
\hline \multicolumn{7}{|c|}{ Shunt Capacitor Limits } & & \\
\hline Bus no & \multicolumn{2}{|l|}{18} & \multicolumn{2}{|c|}{25} & \multicolumn{2}{|l|}{53} & & \\
\hline Qcmin & \multicolumn{2}{|l|}{0} & \multicolumn{2}{|c|}{0} & \multicolumn{2}{|l|}{0} & & \\
\hline Qcmax & \multicolumn{2}{|l|}{10} & \multicolumn{2}{|c|}{5.2} & \multicolumn{2}{|l|}{6.1} & & \\
\hline
\end{tabular}

Table 2: Control variables obtained after optimization

\begin{tabular}{|l|l|}
\hline $\begin{array}{l}\text { Control } \\
\text { Variables }\end{array}$ & ECS \\
\hline V1 & 1.1 \\
\hline V2 & 1.032 \\
\hline V3 & 1.038 \\
\hline V6 & 1.027 \\
\hline V8 & 1.021 \\
\hline V9 & 1.003 \\
\hline V12 & 1.015 \\
\hline Qc18 & 0.0661 \\
\hline Qc25 & 0.200 \\
\hline
\end{tabular}




\begin{tabular}{|l|l|}
\hline Qc53 & 0.0474 \\
\hline T4-18 & 1.001 \\
\hline T21-20 & 1.040 \\
\hline T24-25 & 0.862 \\
\hline T24-26 & 0.873 \\
\hline T7-29 & 1.051 \\
\hline T34-32 & 0.873 \\
\hline T11-41 & 1.019 \\
\hline T15-45 & 1.030 \\
\hline T14-46 & 0.910 \\
\hline T10-51 & 1.020 \\
\hline T13-49 & 1.060 \\
\hline T11-43 & 0.910 \\
\hline T40-56 & 0.900 \\
\hline T39-57 & 0.950 \\
\hline T9-55 & 0.950 \\
\hline
\end{tabular}

Table 3: Comparison results

\begin{tabular}{|l|l|l|l|l|}
\hline S.No. & $\begin{array}{l}\text { Optimization } \\
\text { Algorithm }\end{array}$ & Finest Solution & Poorest Solution & $\begin{array}{l}\text { Normal } \\
\text { Solution }\end{array}$ \\
\hline 1 & NLP [14] & 0.25902 & 0.30854 & 0.27858 \\
\hline 2 & CGA [14] & 0.25244 & 0.27507 & 0.26293 \\
\hline 3 & AGA [14] & 0.24564 & 0.26671 & 0.25127 \\
\hline 4 & PSO-w [14] & 0.24270 & 0.26152 & 0.24725 \\
\hline 5 & PSO-cf [14] & 0.24280 & 0.26032 & 0.24698 \\
\hline 6 & CLPSO [14] & 0.24515 & 0.24780 & 0.24673 \\
\hline 7 & SPSO-07 [14] & 0.24430 & 0.25457 & 0.24752 \\
\hline 8 & L-DE [14] & 0.27812 & 0.41909 & 0.33177 \\
\hline 9 & L-SACP-DE [14] & 0.27915 & 0.36978 & 0.31032 \\
\hline 10 & L-SaDE [14] & 0.24267 & 0.24391 & 0.24311 \\
\hline 11 & SOA [14] & 0.24265 & 0.24280 & 0.24270 \\
\hline 12 & LM [15] & 0.2484 & 0.2922 & 0.2641 \\
\hline 13 & MBEP1 [15] & 0.2474 & 0.2848 & 0.2643 \\
\hline 14 & MBEP2 [15] & 0.2482 & 0.283 & 0.2592 \\
\hline 15 & BES100 [15] & 0.2438 & 0.263 & 0.2541 \\
\hline 16 & BES200 [15] & 0.3417 & 0.2486 & 0.2443 \\
\hline 17 & Proposed ECS & 0.22018 & 0.23072 & 0.22216 \\
\hline
\end{tabular}

Then Enriched Cuckoo Search (ECS) algorithm has been tested in standard IEEE 118-bus test system [16].The system has 54 generator buses, 64 load buses, 186 branches and 9 of them are with the tap setting transformers. The limits of voltage on generator buses are $0.95-1.1$ per-unit., and on load buses are $0.95-1.05$ per-unit. The limit of transformer rate is $0.9-1.1$, with the changes step of 0.025 . The limitations of reactive power source are listed in Table 4 , with the change in step of 0.01 . 
Table 4: Limitation of reactive power sources

\begin{tabular}{|l|l|l|l|l|l|l|l|}
\hline BUS & 5 & 34 & 37 & 44 & 45 & 46 & 48 \\
\hline QCMAX & 0 & 14 & 0 & 10 & 10 & 10 & 15 \\
\hline QCMIN & -40 & 0 & -25 & 0 & 0 & 0 & 0 \\
\hline BUS & 74 & 79 & 82 & 83 & 105 & 107 & 110 \\
\hline QCMAX & 12 & 20 & 20 & 10 & 20 & 6 & 6 \\
\hline QCMIN & 0 & 0 & 0 & 0 & 0 & 0 & 0 \\
\hline
\end{tabular}

The statistical comparison results of 50 trial runs have been list in Table 5 and the results clearly show the better performance of proposed Enriched Cuckoo Search (ECS) algorithm in reducing the real power loss.

Table 5: Comparison results

\begin{tabular}{|l|l|l|l|l|}
\hline Active power loss (MW) & $\begin{array}{l}\text { BBO } \\
{[\mathbf{1 7}]}\end{array}$ & $\begin{array}{l}\text { ILSBBO/ } \\
\text { strategy1 } \\
{[\mathbf{1 7}]}\end{array}$ & $\begin{array}{l}\text { ILSBBO/ } \\
\text { strategy1 } \\
{[\mathbf{1 7 ]}}\end{array}$ & $\begin{array}{l}\text { Proposed } \\
\text { ECS }\end{array}$ \\
\hline Min & 128.77 & 126.98 & 124.78 & 117.42 \\
\hline Max & 132.64 & 137.34 & 132.39 & 121.06 \\
\hline Average & 130.21 & 130.37 & 129.22 & 118.74 \\
\hline
\end{tabular}

\section{Conclusion}

This paper successfully solves optimal reactive power problem by Enriched Cuckoo Search (ECS) algorithm. Due to the parameters of the cuckoo search algorithm has been kept constant \& it decreases the efficiency of the algorithm. In this paper cuckoo search algorithm parameters pa ,$\lambda$ and $\alpha$ are tuned to find improved solutions. In order to evaluate the performance of the proposed algorithm, it has been tested in Standard IEEE 57,118 bus systems and compared to other standard reported algorithms. Simulation results show's that Enriched Cuckoo Search (ECS) algorithm successfully reduces the real power loss and voltage profiles are within the limits.

\section{References}

[1] O.Alsac, and B. Scott, "Optimal load flow with steady state security",IEEE Transaction. PAS 1973, pp. 745-751.

[2] Lee K Y ,Paru Y M , Oritz J L -A united approach to optimal real and reactive power dispatch , IEEE Transactions on power Apparatus and systems 1985: PAS-104 : 1147-1153

[3] A.Monticelli, M .V.F Pereira ,and S. Granville , "Security constrained optimal power flow with post contingency corrective rescheduling", IEEE Transactions on Power Systems :PWRS-2, No. 1, pp.175-182.,1987.

[4] Deeb N ,Shahidehpur S.M ,Linear reactive power optimization in a large power network using the decomposition approach. IEEE Transactions on power system 1990: 5(2) : 428-435

[5] E. Hobson ,'Network consrained reactive power control using linear programming, ' IEEE Transactions on power systems PAS -99 (4) ,pp 868=877, 1980

[6] K.Y Lee ,Y.M Park, and J.L Oritz, "Fuel -cost optimization for both real and reactive power dispatches", IEE Proc; 131C,(3), pp.85-93.

[7] M.K. Mangoli, and K.Y. Lee, "Optimal real and reactive power control using linear programming”, Electr.Power Syst.Res, Vol.26, pp.1-10,1993. 
[8] K.Anburaja, “Optimal power flow using refined genetic algorithm”, Electr.Power Compon.Syst , Vol. 30, 1055-1063,2002.

[9] D. Devaraj, and B. Yeganarayana, "Genetic algorithm based optimal power flow for security enhancement”, IEE proc-Generation.Transmission and. Distribution; 152, 6 November 2005.

[10] Payne R B, Sorenson M D, Klitz K (2005) The cuckoos. Oxford University Press.

[11] Yang XS, Deb S (2009) Cuckoo search via Lévy flights, In: Proceeings of World Congress on Nature \& Biologically Inspired Computing (NaBIC 2009, India), 210-214.

[12] Yang XS, Deb S (2010) Engineering Optimisation by Cuckoo Search, Int. J. Mathematical Modelling and Numerical Optimisation, 1(4):330-343.

[13] Brown C, Liebovitch LS, Glendon R (2007) Lévy flights in Dobe Ju/hoansi foraging patterns, Hum Ecol. 35:129-138.

[14] Chaohua Dai, Weirong Chen, Yunfang Zhu, and Xuexia Zhang, "Seeker optimization algorithm for optimal reactive power dispatch,” IEEE Trans. Power Systems, Vol. 24, No. 3, August 2009, pp. 1218-1231.

[15] J. R. Gomes and 0. R. Saavedra, "Optimal reactive power dispatch using evolutionary computation: Extended algorithms," IEE Proc.-Gener. Transm. Distrib.. Vol. 146, No. 6. Nov. 1999.

[16] IEEE, "The IEEE 30-bus test system and the IEEE 118-test system”, (1993), http://www.ee.washington.edu/trsearch/pstca/.

[17] Jiangtao Cao, Fuli Wang and Ping Li, "An Improved Biogeography-based Optimization Algorithm for Optimal Reactive Power Flow" International Journal of Control and Automation Vol.7, No.3 (2014), pp.161-176.

*Corresponding author.

E-mail address: gklenin@ gmail.com 